\title{
Laboratory evaluation of a flow cytometric BCR-ABL immunobead assay
}

\author{
Zsuzsanna Hevessy ${ }^{1}$, Renáta Hudák ${ }^{1}$, \\ Valéria Kiss-Sziráki ${ }^{1}$, Péter Antal-Szalmás ${ }^{1}$, \\ Miklós Udvardy², László Rejto ${ }^{2}$, László Szerafin ${ }^{3}$ \\ and János Kappelmayer ${ }^{1, *}$ \\ ${ }^{1}$ Department of Laboratory Medicine, Medical and Health \\ Science Center, University of Debrecen, Debrecen, Hungary \\ ${ }^{2}$ Department of Medicine, Medical and Health Science \\ Center, University of Debrecen, Debrecen, Hungary \\ ${ }^{3}$ Department of Hematology, Jósa András Teaching Hospital, \\ Nyíregyháza, Hungary
}

\begin{abstract}
Background: A new flow cytometric (FC) BCR-ABL immunobead assay has been developed recently. Here we present the laboratory evaluation of the commercially available kit. Methods: Mononuclear cells were isolated, lysed and processed according to the instructions of the manufacturer. Anti-BCR antibodies adsorbed to capture beads bind the BCR-ABL fusion proteins of the lysed cells, a phycoerythrin (PE)-conjugated anti-ABL antibody is the detector reagent and mean fluorescence intensity (MFI) signals were recorded by flow cytometry. Detection of $\mathrm{t}(9 ; 22)(\mathrm{q} 34 ; \mathrm{q} 11)$ translocation was carried out with a quantitative PCR assay.

Results: MFI results of 20 normal peripheral blood samples were $88 \pm 8$ (mean \pm SD), CV 9\%. K562 cells were used as positive control. Within-batch imprecision was excellent $(3.7 \%$ in the normal and $10 \%$ in the pathological range). Cut-off was chosen at MFI 112, where both sensitivity and specificity were $100 \%$. Altogether 17 chronic myeloid leukemia (CML) and 16 acute leukemia samples were analyzed. All PCR positive samples $(n=14)$ were positive with the FC method and negative results were also concordant $(n=15)$. Frozen cell lysates can be stored up to 4 weeks without significant decrease of MFI signal.

Conclusions: The FC BCR-ABL assay is a fast, reproducible and reliable method that may be incorporated into standard flow cytometric protocols to help clinical decision-making.
\end{abstract}

\footnotetext{
*Corresponding author: János Kappelmayer, Department of Laboratory Medicine, Medical and Health Science Center, University of Debrecen, 4032 Debrecen, Nagyerdei krt. 98, Hungary

Phone: +3652 340006, Fax: +3652 417631,

E-mail: kappelmayer@med.unideb.hu

Received September 8, 2011; accepted November 30, 2011
}

Keywords: BCR-ABL; flow cytometry; immunobead assay.

The $B C R-A B L$ fusion gene can be detected in chronic myeloid leukemia (CML), in $2 \%-5 \%$ of childhood and $30 \%-35 \%$ of adult acute lymphoblastic leukemia (ALL) cases (1). Introduction of tyrosine kinase inhibitors (TKI) into the treatment of CML and Ph+ ALL have profoundly changed the prognosis of these diseases (2-4). In adult patients diagnosed with $\mathrm{Ph}+$ ALL, chemotherapy combined with TKI represents the current standard of care with newly diagnosed disease. This makes detection of $B C R-A B L$ aberrations of utmost importance.

Detection of $\mathrm{t}(9 ; 22)$ is generally carried out at the chromosome level using karyotyping or fluorescence in situ hibridization (FISH) or at the mRNA level using PCR techniques. These methods require specialized laboratories and welltrained personnel, furthermore they are time-consuming. The turn-around-time (TAT) for FISH and PCR techniques is 2-3 days while that of karyotyping is 1-2 weeks. Soon after the first attempt to detect chromosome translocations with beadbased flow cytometry (5) a new, rapid method has been developed to detect the BCR-ABL oncoprotein (6).

While the $A B L$ gene contains one large breakpoint region, three breakpoint regions [major breakpoint cluster region (M-bcr), minor breakpoint cluster region (m-bcr) and micro breakpoint cluster region $(\mu-b c r)]$ and several additional rare breakpoints have been found in the $B C R$ gene. As a result several different fusion transcripts and fusion proteins can be formed. The immunoassay was developed to detect all possible variants of the BCR-ABL oncoprotein. The BCRABL Protein Kit was first tested in a multicenter study on 145 BM or PB samples of patients suspected to have CML or acute leukemia and PB of 72 healthy individuals. In this study Weerkamp et al. found full concordance between the PCR and the Protein Kit results when a mean fluorescence intensity (MFI) cut-off of 135 was chosen (6). Raponi et al. investigated 101 samples of acute leukemia patients and concluded that PCR and FC results were concordant in all de novo cases but two patients who were pretreated with steroid and had $<10 \%$ blasts showed negative FC and positive PCR results (7). Recently D'Alessio et al. described the miniaturization of the FC immunobead assay and detected the BCR-ABL protein in cerebrospinal fluid (8). Cools and Vandenberghe suggested incorporating the new FC test into a diagnostic algorithm of hematological malignancies (9). In the near future several other bead-based immunoassays will be developed in order to detect fusion oncoproteins in a few set in italic - please confirm that italic font has been applied correctly throughout the article. 
hours and fulfil clinical needs according to modern standards $(10,11)$.

The aim of this study was to evaluate the imprecision of the new test in normal and pathological ranges and to determine the cut-off MFI that dicriminates between PCR positive and negative samples. We also determined diagnostic sensitivity and specificity, as well as the stability of the positive signal in cell lysates over 5 months.

EDTA-anticoagulated peripheral blood (PB) of 20 healthy volunteers and $\mathrm{PB}$ and bone marrow (BM) aliquots from the remnant of the diagnostic sample of 33 hematological patients (17 with CML and 16 with acute leukemia) were investigated. Mononuclear cells were separated and a standardized amount of cells $\left(2.5 \times 10^{6}\right)$ was lysed and processed according to the instructions of BCR-ABL Protein Kit (Becton Dickinson, San Jose, CA, USA). Anti-BCR antibodies adsorbed to capture beads bind the BCR-ABL fusion proteins and a phycoerythrin (PE)-conjugated anti-ABL antibody is the detector reagent (6).

To enable comparison between different days, BD FACSCanto II flow cytometer was calibrated daily using Calibrite fluorescent microbeads (Cat No. 641,319, Becton Dickinson, San Jose, CA, USA) and Autocomp software as recommended by the manufacturer. After the capture beads have been identified and gated on the basis of their scatter and fluorescence characteristics, the presence of sandwich complexes could be detected by PE (FL2) MFI signals generated by the detector reagent. Data were recorded and analyzed with a FACSDiva software (Becton Dickinson, San Jose, CA, USA). Samples lacking the BCR-ABL fusion protein gave a negative signal (Figure 1A) while K562 cell line, containing the oncoprotein gave intensely positive signal (Figure 1B).

Detection of $\mathrm{t}(9 ; 22)(\mathrm{q} 34 ; \mathrm{q} 11)$ translocation was carried out with a quantitative PCR assay [Light Cycler $\mathrm{t}(9 ; 22)$ Quantification Kit, Roche Diagnostics, Basel, Switzerland] (12) standardized internationally in the EUTOS program. Thirteen out of 14 PCR positive samples were analyzed with nested PCR according to a standard protocol (13).

A
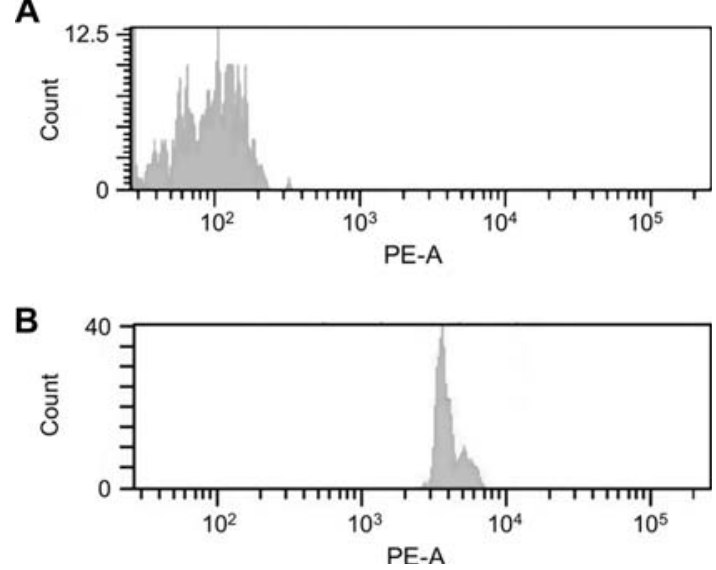

Figure 1 Representative histograms of a negative and a positive sample.

(A) Samples lacking the BCR-ABL fusion protein resulted in a negative signal while (B) K562 cell line, containing the oncoprotein gave positive signal.
Cell lysates of pooled normal WBC and K562 cells diluted to have MFI value slightly above cut-off (MFI 138), another with MFI 500 and four patient's peripheral blood mononuclear cells (PBMC) were stored at $-80^{\circ} \mathrm{C}$ and thawed after 1 day $(n=5) ; 3$ days $(n=2), 1$ week $(n=1) ; 4$ weeks $(n=8)$; 16 weeks $(n=1)$ and 20 weeks $(n=3)$. Statistical analysis was performed with GraphPad Prism 4.0 software.

Lysates from normal peripheral leukocytes (WBC) and from the K562 cell line were used as negative and positive control, respectively. Mean PE (FL2) MFI of 20 normal PB samples processed and measured on 20 consecutive days was $88 \pm 8$ (mean $\pm \mathrm{SD}$ ), between-run precision $\mathrm{CV}$ was $9 \%$. Within-run precision was carried out on a pool of normal PB samples isolated in one step, then lysed and labeled separately. Eight samples were processed and acquired, with $3.7 \% \mathrm{CV}$. Within-run precision in the pathological range was investigated on K562 cell lysates $(n=6)$, mean MFI 3958, SD 397, CV $10 \%$.

Altogether $17 \mathrm{CML}$ and 16 acute leukemia patient samples (PB and $\mathrm{BM}$ ) of de novo cases were analyzed. Among acute leukemia samples 14 were cALL, one de novo AML and one AML transformed from CML. All PCR positive samples $(n=14)$ were found to be positive with the FC method and negative results were also concordant $(n=15)$ (Figure 2A). In the positive samples nested PCR detected six cases with b2a2, five cases with b3a2 and two cases with e1a2 rearrangement, while nested PCR was not performed in one cALL sample. MFI of the positive samples ranged from 118 to 4905 but no correlation could be demonstrated between the MFI values and the quantitative PCR results (data not shown). Parallel examination of $\mathrm{PB}$ and $\mathrm{BM}$ samples (in four patients) showed concordant results in the FC test. In these four cases PCR was performed only from $\mathrm{PB}$.

Performing a ROC analysis area under the curve was 1.0, sensitivity and specificity of the test was $100 \%$ (CI 95\%) when the cut-off was set at MFI 112, which was the mean MFI+3SD of 20 normal WBC samples. As traditional ROC curve was definitely a $90^{\circ}$-broken line, we demonstrated the determination of the cut-off MFI, sensitivity and specificity on one graph (Figure 2B). It shows that lowering the cut-off to MFI 95 resulted in $60 \%$ specificity and elevating it to MFI 703 decreased the sensitivity to $57 \%$.

Investigation of the stability of fusion protein in frozen cell lysates showed no significant loss of the positive signal slightly above cut-off level. After 4 weeks fluorescent signal decreased by $1.5 \%$, even after 20 weeks it decreased by $5.8 \%$. The higher the MFI of the fresh K562 lysate the greater loss of MFI could be detected. When MFI was 500 of the fresh sample, $15 \%$ signal loss was detected after 4 weeks and $28 \%$ after 20 weeks (Figure 2C). The same phenomenon could be observed in case of patient samples. No loss of the fluorescent signal was detected in the negative cases while $38 \%$ and $43 \%$ MFI loss was found in the positive cases, as the MFI value of their fresh sample was very high [737, 3212]. Based on these results we recommend storing frozen cell lysates for not more than 4 weeks.

In conclusion, the flow cytometric BCR-ABL assay can detect all forms of the BCR-ABL fusion protein in $4 \mathrm{~h}$ with 
A

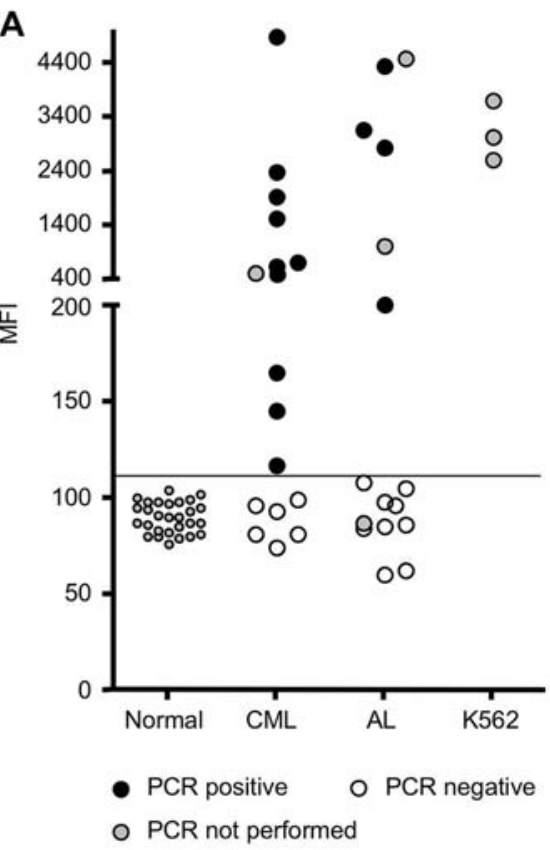

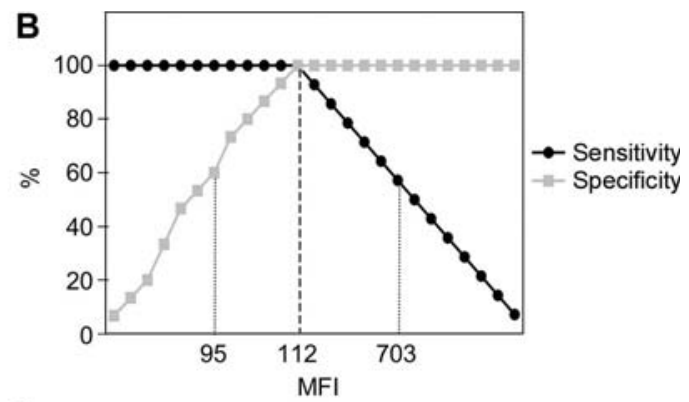

C

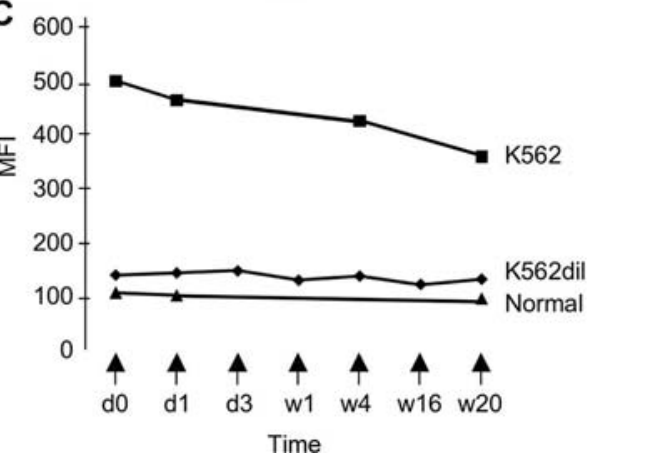

Figure 2 Laboratory evaluation of the flow cytometric BCR-ABL bead-based immunoassay.

(A) Assay results of different sample groups (normal, chronic myeloid leukemia and acute leukemia patients, K562 cells). Mean fluorescence intensity (MFI) is shown on $y$-axis, horizontal line indicates cut-off at MFI 112. Black-filled circles indicate positive, open circle negative result with a quantitative PCR method, in case of gray-filled circles PCR was not performed. (B) Sensitivity and specificity of the test was $100 \%$ when cut-off was at MFI 112. Lowering cut-off level decreased specificity, while elevating it decreased sensitivity. (C) Investigation of the stability of fusion protein in frozen cell lysates showed no significant loss of the positive signal slightly above cut-off level (K562dil). The higher the MFI of the fresh K562 lysate the greater loss of MFI could be detected. When MFI was 500 of the fresh sample, 15\% signal loss was detected after 4 weeks and $28 \%$ after 20 weeks.

high sensitivity and specificity. In our study in the FC positive samples which were tested with nested PCR the three most frequent rearrangement types were found. Cut-off should be determined in each laboratory as the mean MFI+3SD of 20 normal WBC lysates. Frozen cell lysates can be stored up to 4 weeks without the decrease of fluorescent signal.

Flow cytometry has long been a hallmark of the diagnosis of hematological malignancies, mainly to identify the malignant clone and characterize it by the detection of surface and intracellular proteins. In a diagnostic setting the short turnaround time, ease and specificity of the new BCR-ABL FC assay makes it a candidate to be part of an algorithm of investigation of de novo, untreated patients. Based on the result of the FC assay PCR and FISH techniques can be rationally triggered. Moreover, it may serve as a molecular diagnosis in geographical areas where PCR technique is not available. This rapid, simple and reliable flow cytometric method opens a novel targeted diagnostic and therapeutic approach for the management of $\mathrm{Ph}^{+}$ALL patients. We recommend its incorporation into standard flow cytometric protocols in order to help clinical decision-making.

\section{Acknowledgments}

This study was supported by the János Bolyai Research Scholarship of the Hungarian Academy of Sciences and the TÁMOP 4.2.1./
B-09/1/KONV-2010-0007 and TÁMOP 4.2.2/V-10/1-2010-0024 project.

\section{Conflict of interest statement}

Authors' conflict of interest disclosure: The authors stated that there are no conflicts of interest regarding the publication of this article. Research support played no role in the study design; in the collection, analysis, and interpretation of data; in the writing of the report; or in the decision to submit the report for publication.

Research funding: None declared.

Employment or leadership: None declared.

Honorarium: None declared.

\section{References}

1. Rowley JD. Chromosomal translocations: revisited yet again. Blood 2008;112:2183-9.

2. Druker BJ, Talpaz M, Resta DJ, Peng B, Buchdunger E, Ford JM, et al. Efficacy and safety of a specific inhibitor of the BCRABL tyrosine kinase in chronic myeloid leukemia. N Engl J Med 2001;344:1031-7.

3. Mathisen MS, O'Brien S, Thomas D, Cortes J, Kantarjian H, Ravandi F. Role of tyrosine kinase inhibitors in the management of Philadelphia chromosome-positive acute lymphoblastic leukemia. Curr Hematol Malig Rep 2011;6:187-94. 
4. Lee HJ, Thomson JE, Wang ES, Wetzler M. Philagelphia chromosome-positive acute lymphoblastic leukemia. Current treatment and future perspectives. Cancer 2011;117:1583-94.

5. Chan HE, Jilani I, Chang R, Albitar M. Detection of chromosome translocations by bead-based flow cytometry. Methods Mol Biol 2007;378:167-74.

6. Weerkamp F, Dekking E, Ng YY, van der Velden VH, Wai H, Böttcher S, et al. Flow cytometric immunobead assay for the detection of BCR-ABL fusion proteins in leukemia patients. Leukemia 2009;23:1106-17.

7. Raponi S, De Propris MS, Wai H, Intoppa S, Elia L, Diverio D, et al. An accurate and rapid flow cytometric diagnosis of BCRABL positive acute lymphoblastic leukemia. Haematologica 2009;94:1767-70.

8. D’Alessio F, Mirabelli P, Mariotti E, Raia M, Di Noto R, Fortunato G, et al. Miniaturized flow cytometry-based BCR-ABL immunoassay in detecting leptomeningeal disease. Leuk Res 2011;35:1290-3.

9. Cools J, Vandenberghe P. New flow cytometry in hematologic malignancies. Haematologica 2009;94:1639-41.
10. Dekking E, van der Velden VH, Böttcher S, Brüggemann M, Sonneveld E, Koning-Goedheer A, et al. Detection of fusion genes at the protein level in leukemia patients via the flow cytometric immunobead assay. Best Pract Res Clin Haematol 2010;23:333-45.

11. Dekking EH, van der Velden VH, Varro R, Wai H, Böttcher S, Kneba M, et al. Flow cytometric immunobead assay for fast and easy detection of PML-RARA fusion proteins for the diagnosis of acute promyelocytic leukemia. Leukemia (in press).
Q2:

Please update ref. 11
12. Lu X, Song X, Ye Y, Liu X, Zhou Y, Zhang L, et al. Quantitative detection of BCR-ABL fusion gene and its application in monitoring chronic myeloid leukemia treatment. Mol Biol Rep 2011;38:3101-5.

13. van Dongen JJ, Macintyre EA, Gabert JA, Delabesse E, Rossi V, Saglio G, et al. Standardized RT-PCR analysis of fusion gene transcripts from chromosome aberrations in acute leukemia for detection of minimal residual disease. Report of the BIOMED-1 Concerted Action: investigation of minimal residual disease in acute leukemia. Leukemia 1999;13:1901-28. 